Bio - grafía. Escritos sobre la Biología y su Enseñanza. ISSN 2027-1034

Edición Extraordinaria. p.p. 1026 - 1033

Memorias del IX Encuentro Nacional de Experiencias en Enseñanza de la Biología y la Educación Ambiental. IV Congreso Nacional de Investigación en Enseñanza de la Biología.

\title{
ANÁLISIS DE LAS IDEAS PREVIAS ACERCA DEL SISTEMA NERVIOSO EN ESTUDIANTES DE GRADO OCTAVO
}

ANALYSIS OF PREVIOUS IDEAS ABOUT THE NERVOUS SYSTEM IN EIGHTH GRADE STUDENTS

\author{
Cristina Villabona Orozco ${ }^{1}$ \\ Alejandra Herrán Urrea ${ }^{2}$
}

\section{RESUMEN}

El conocimiento adquirido a través de las experiencias personales, de la cotidianidad y del contexto en el que se desarrollan los alumnos siempre estará conectado ineludiblemente a la interpretación que estos dan a lo enseñado dentro del aula en las clases de ciencias naturales. Las ideas previas acerca del Sistema Nervioso se pueden analizar desde la comprensión que tienen los estudiantes acerca de su morfología, su funcionamiento, la integración con el resto del cuerpo y las respuestas que produce en contacto con el medio externo. Para este trabajo se analizaron las ideas previas acerca del sistema nervioso en estudiantes del grado octavo (802) en la I.E.D Alejandro Obregón, Bogotá, D.C. La metodología utilizada se enmarca dentro del paradigma cualitativo interpretativo, utilizando como instrumento de análisis una caracterización de ideas previas por medio de una serie de preguntas escritas. Para el análisis se postularon cuatro categorías utilizadas para la sistematización de las ideas previas por Beltrán y Castro (2016). Dentro de los resultados se analiza que los estudiantes reconocen ciertas características del sistema nervioso, aunque por lo general se observa que aíslan el sistema nervioso de los otros sistemas del cuerpo, se desconoce la unidad básica que lo integra, la neurona y su función, en muchos casos reconocen algunas funciones pero no logran explicarlas o asociarlas con su entorno.

PALABRAS CLAVES: Ideas previas, Sistema nervioso, aprendizaje, cotidianidad, explicación.

\footnotetext{
${ }^{1}$ Estudiante de Licenciatura en Biología, Universidad Distrital Francisco José de Caldas, Bogotá, Colombia, contacto: cristinavillabona3@gmail.com-3057234752

${ }^{2}$ Estudiante de Licenciatura en Biología, Universidad Distrital Francisco José de Caldas, Bogotá, Colombia, contacto: aleherran.18@gmail.com - 3133088431
} 
Bio - grafía. Escritos sobre la Biología y su Enseñanza. ISSN 2027-1034

Edición Extraordinaria. p.p. 1026 - 1033

Memorias del IX Encuentro Nacional de Experiencias en Enseñanza de la Biología y la

Educación Ambiental. IV Congreso Nacional de Investigación en Enseñanza de la

Biología.

\begin{abstract}
The knowledge acquired through personal experiences, daily life and the context in which students develop will always be inextricably linked to the interpretation they give to what is taught within the classroom in natural science classes. Previous ideas about the Nervous System can be analyzed from students' understanding of their morphology, functioning, integration with the rest of the body and the responses it produces in contact with the external environment. For this work the previous ideas about the nervous system were analyzed in eighth grade students (802) in the I.E.D Alejandro Obregón, Bogotá, D.C. The methodology used is framed within the interpretive qualitative paradigm, using as an analysis instrument a characterization of previous ideas through a series of written questions. For the analysis, four categories used for the systematization of the previous ideas by Beltrán and Castro (2016) were postulated. Within the results it is analyzed that the students recognize certain characteristics of the nervous system, although generally it is observed that they isolate the nervous system of the other systems of the body, it is not known the basic unit that integrates it, the neuron and its function, in Many cases recognize some functions but fail to explain them or associate them with their environment.
\end{abstract}

KEY WORDS: Previous ideas, nervous system, learning, everyday, explanation.

\title{
INTRODUCCIÓN
}

Entender que el conocimiento escolar no se construye sólo en el aula sino también en la cotidianidad del estudiante permitirá vincular las ideas previas de los estudiantes a la complejización del conocimiento y a la asimilación de contenidos. Estas ideas de los alumnos constituyen un referente fundamental para la construcción de un currículo escolar alternativo, socialmente más pertinente, que permita a la escuela participar efectivamente en la construcción de las sociedades del siglo XXI (González \& GarcíaPérez, 2014).

Las ideas que tienen los estudiantes y que en ocasiones de manera equivocada se consideran "erróneas", o que se constituyen como obstáculos y con las cuales los docentes muchas veces no encuentran un acuerdo, podrían ser consideradas dentro del proceso de enseñanza-aprendizaje como un elemento más de la formación, entendido así; estas no deben tratarse como problemas diferentes que hay que enfrentar por separado, sino como diferentes manifestaciones de un mismo problema, la desconexión entre el conocimiento que los estudiantes generan para dar sentido al mundo que les rodea, un mundo de objetos y personas, y el conocimiento científico, plagado de extraños símbolos y conceptos abstractos referidos a un mundo más imaginario que real (Pozo, 
Bio - grafía. Escritos sobre la Biología y su Enseñanza. ISSN 2027-1034

Edición Extraordinaria. p.p. 1026 - 1033

Memorias del IX Encuentro Nacional de Experiencias en Enseñanza de la Biología y la

Educación Ambiental. IV Congreso Nacional de Investigación en Enseñanza de la Biología.

1996).

Para abordar entonces las ideas previas que tienen los estudiantes sobre el Sistema Nervioso es importante comprender su morfología, su funcionamiento y en general la gran complejidad de reacciones de control que puede llevar a cabo, pues recibe literalmente miles de datos e información procedentes de los diferentes órganos sensoriales que se encuentran en interacción con el medio, para luego integrarlos, interpretarlos y dar respuestas a partir de reacciones sensitivas y motoras del cuerpo (Guyton, 1978). Olave et al (2015) y Salazar (2013) mencionan que los estudiantes de básica secundaria intentan explicar el concepto de SN por su utilidad, reduciéndolo así a una de sus cualidades; En algunos casos suelen mencionar algunas partes relacionadas con el mismo, sin embargo desconocen la función y ubicación. Términos como reacción, estímulo, coordinación son utilizadas por los estudiantes sin la debida explicación lo que puede llevar a equivocaciones ya que se omiten detalles que permiten que el concepto tenga sentido y validez científica.

Teniendo en cuenta estos referentes conceptuales el objetivo de esta investigación es reconocer las ideas previas de los estudiantes sobre el SN, a través de categorías que nos permiten identificar la coherencia en las explicaciones de los fenómenos postulados.

\section{METODOLOGÍA}

La investigación cualitativa es interpretativa, multimetódica, reflexiva, profunda y rigurosa (Denzin \& Lincoln, 1994) permitiendo establecer un análisis disciplinado acerca de los conocimientos previos que poseen los estudiantes. El análisis de las ideas previas de los estudiantes sobre el sistema nervioso se realizó en dos fases: en la primera, se implementó un instrumento con 6 preguntas y se realizó un análisis de cada una estas; en la segunda se categorizaron estas respuestas utilizando el modelo propuesto por Beltrán y castro (2016); las cuales se explican en la siguiente tabla (Tabla 1).

\begin{tabular}{|l|l|}
\hline Categoría & Definición \\
\hline $\begin{array}{l}\text { 1. Relaciona de manera clara el nivel de } \\
\text { organización del SN y su importancia } \\
\text { dentro de los organismos. }\end{array}$ & $\begin{array}{l}\text { Respuestas acertadas constituidas con una } \\
\text { explicación clara y concisa del fenómeno } \\
\text { postulado. }\end{array}$ \\
\hline $\begin{array}{l}\text { 2. Manifiesta obstáculos que no le } \\
\text { permiten dar explicaciones sobre la la }\end{array}$ & $\begin{array}{l}\text { Respuesta más o menos correctas, pero sin } \\
\text { claridad alguna, en el momento de justificarlas } \\
\text { pueden estar mal redactadas y no existe }\end{array}$ \\
\hline
\end{tabular}


Bio - grafía. Escritos sobre la Biología y su Enseñanza. ISSN 2027-1034

Edición Extraordinaria. p.p. 1026 - 1033

Memorias del IX Encuentro Nacional de Experiencias en Enseñanza de la Biología y la Educación Ambiental. IV Congreso Nacional de Investigación en Enseñanza de la Biología.

\begin{tabular}{|l|l|} 
funcionalidad e importancia del SN & coherencia. \\
\hline $\begin{array}{l}\text { 3. Sin conocimiento previo alguno acerca } \\
\text { de los elementos biológicos que se } \\
\text { relacionan con el SN }\end{array}$ & $\begin{array}{l}\text { Respuestas y explicaciones a los fenómenos } \\
\text { propuestos con un alto grado de desconexión } \\
\text { e incoherencia. }\end{array}$ \\
\hline 4. No sabe/ no responde & Respuestas en blanco \\
\hline
\end{tabular}

El instrumento de ideas previas fue implementado en 36 estudiantes del curso 802 del I.E.D Alejandro Obregón, en la localidad Rafael Uribe Uribe, Bogotá D.C., cuyas edades oscilan entre los 12 y 15 años de edad. El diseño de las preguntas se realizó teniendo en cuenta la edad, grado y aspectos acerca del tema de sistema nervioso tratando de indagar las ideas presentes sobre aspectos como: morfología, funcionalidad y asociación con su propio entorno.

\section{RESULTADOS}

A continuación se encuentran las preguntas realizadas en el instrumento de ideas previas y su respectivo análisis.

1. ¿Cuál es la función del sistema nervioso?

En general las respuestas de los estudiantes se remitieron al control de todas las acciones realizadas por el cuerpo, en las que se incluyen pensar, moverse, procesar y/o ordenar información, y dar respuesta a ciertos estímulos. Uno de los estudiantes lo define como una red de tejidos que transmiten información a través de neuronas.

2. ¿Tienen las plantas sistema nervioso?

Algunos estudiantes consideran que las plantas carecen de $\mathrm{SN}$ debido principalmente a la ausencia de movimiento, sin embargo, para algunos las plantas si lo presentan, ya que gracias a él, ellas pueden realizar fotosíntesis para obtener su propio alimento, y responder a estímulos que les permiten reproducirse y crecer, además la existencia de la savia bruta es una prueba de que tal sistema está presente en la planta.

3. ¿Quién regula la función del corazón y la respiración?

La mayoría de los estudiantes considera que el cerebro es el que cumple dicha función, 
Edición Extraordinaria. p.p. 1026 - 1033

Memorias del IX Encuentro Nacional de Experiencias en Enseñanza de la Biología y la Educación Ambiental. IV Congreso Nacional de Investigación en Enseñanza de la Biología.

otros consideran que es gracias al cerebro y a los pulmones. Un pequeño grupo lo atribuye específicamente a los pulmones o el sistema respiratorio, y 2 estudiantes señalan al sistema circulatorio debido al transporte de oxígeno que permitiría que estas funciones se den. Por último, algunos estudiantes mencionan que esta función no es regulada por ningún sistema, debido a que son músculos involuntarios.

4. ¿Qué es una neurona y cuáles son sus partes?

Ninguno de los estudiantes logró esquematizar la neurona y sus partes, pero todos la definieron como una célula o estructura que les permite pensar, transmitir señales, producir y almacenar información.

5. ¿Por qué se dan los reflejos?

Muy pocos estudiantes contestaron esta respuesta, y mencionaba que era una respuesta del cuerpo o del cerebro para escapar o evadir alguna situación, además mencionaron que podría también ser un proceso de intuición o un movimiento involuntario.

6. ¿Por qué nos da miedo?

Está respuesta fue la que mayor dificultad presentó en los estudiantes, la gran mayoría no la respondió y los que lograron hacerla consideran que se da cuando una persona tiene traumas, fobias, temores pero ninguno lo asoció con procesos del sistema nervioso.

\section{DISCUSIÓN}

La siguiente tabla contiene la categorización de cada una de las respuestas dadas por los estudiantes (Tabla 2).

\begin{tabular}{|l|c|c|c|c|c|c|c|l|}
\hline Pregunta & \multicolumn{2}{|l|}{ Categoría 1 } & \multicolumn{2}{l|}{ Categoría 2 } & \multicolumn{2}{l|}{ Categoría 3 } & \multicolumn{2}{l|}{ Categoría 4 } \\
\hline & $\begin{array}{l}\text { N. de de } \\
\text { estudia } \\
\text { ntes }\end{array}$ & $\begin{array}{l}\text { Porcen } \\
\text { taje }\end{array}$ & $\begin{array}{l}\text { N. de } \\
\text { estudia } \\
\text { ntes }\end{array}$ & $\begin{array}{l}\text { Porcen } \\
\text { taje }\end{array}$ & $\begin{array}{l}\text { N. de } \\
\text { estudia } \\
\text { ntes }\end{array}$ & $\begin{array}{l}\text { Porcentaj } \\
\text { e }\end{array}$ & $\begin{array}{l}\text { N. de } \\
\text { estudia } \\
\text { ntes }\end{array}$ & $\begin{array}{l}\text { Porcen } \\
\text { taje }\end{array}$ \\
\hline 1 & 6 & $16,6 \%$ & 20 & $55,5 \%$ & 10 & $27,7 \%$ & 0 & $0 \%$ \\
\hline
\end{tabular}


Bio - grafía. Escritos sobre la Biología y su Enseñanza. ISSN 2027-1034

Edición Extraordinaria. p.p. 1026 - 1033

Memorias del IX Encuentro Nacional de Experiencias en Enseñanza de la Biología y la Educación Ambiental. IV Congreso Nacional de Investigación en Enseñanza de la Biología.

\begin{tabular}{|l|c|c|c|c|c|c|c|c|} 
& & & & & & & & \\
\hline 2 & 0 & $0 \%$ & 14 & $38,8 \%$ & 19 & $52,7 \%$ & 3 & $8,3 \%$ \\
\hline 3 & 14 & $38,8 \%$ & 7 & $19,4 \%$ & 14 & $38,8 \%$ & 1 & $2,7 \%$ \\
\hline 4 & 0 & $0 \%$ & 7 & $19,4 \%$ & 25 & $69,4 \%$ & 4 & $11,1 \%$ \\
\hline 5 & 0 & $0 \%$ & 3 & $8,3 \%$ & 27 & $75 \%$ & 6 & $16,6 \%$ \\
\hline 6 & 0 & $0 \%$ & 2 & $5,5 \%$ & 19 & $52,7 \%$ & 15 & $41,6 \%$ \\
\hline
\end{tabular}

El reconocimiento de las ideas previas que presentan los estudiantes nos permite empezar a trabajar sobre una temática en particular, que en este caso es el sistema nervioso. Es importante identificar las ideas previas que los estudiantes traen a clase, Ausubel (1983) menciona "como factor que incide en el aprendizaje aquello que el aprendiz ya sabe, averígüese esto y enséñese de acuerdo a ello". Moreira (1993) amplía esta frase diciendo "aquello que el aprendiz ya sabe" se refiere a la "estructura cognitiva" el contenido total y la organización de sus ideas en esa área particular del conocimiento.

Las ideas previas que presentan los estudiantes del curso 802, carecen de una adecuada justificación, en muchos casos reconocen algunas funciones pero no logran explicarlas o asociarlas con su entorno, ejemplo de esto, es que la mayoría no respondió la pregunta ¿por qué sentimos miedo?; Comparando con trabajos anteriores hay congruencias en algunas nociones como que el Sistema Nervioso y el cerebro son independientes, que este se asocia más con los animales que con las plantas y se traduce en una serie de comportamientos como: sentir dolor, correr, pensar, se asocia más a los animales vertebrados que a los invertebrados, (Serrano, 1988; citado por Luna \& Solis, 1997). Los estudiantes tienden a desligar los órganos que lo integran, dejando el cerebro como un todo (Olave et al, 2015).

Este trabajo de caracterización de las ideas previas de los estudiantes y sus resultados, no generaliza que dichas sean la totalidad de las concepciones que manejan los estudiantes, por el tiempo usado para la actividad y por la disposición de los estudiantes para responderla. 
Bio - grafía. Escritos sobre la Biología y su Enseñanza. ISSN 2027-1034

Edición Extraordinaria. p.p. 1026 - 1033

Memorias del IX Encuentro Nacional de Experiencias en Enseñanza de la Biología y la

Educación Ambiental. IV Congreso Nacional de Investigación en Enseñanza de la Biología.

\section{CONCLUSIONES}

-Comprender los conocimientos previos acerca del SN en estudiantes de secundaria permitirá crear e implementar nuevas metodologías o formas de enseñanza para lograr un aprendizaje significativo en los estudiantes.

-Se analiza un porcentaje considerablemente alto de estudiantes que se encuentran en la categoría número 3, pero el porcentaje es más bajo en la última categoría exceptuando la pregunta seis, observando entonces que hay dificultades en cuanto al conocimiento previo acerca de algunos aspectos del SN, pero no se presenta un desconocimiento total.

-Se observa como en la medida en que la pregunta se complejiza (pregunta \#6) y da cuenta de la relación con el medio y su propio cuerpo, los estudiantes presentan más dificultades. Por ello analizar las ideas previas de los estudiantes y apoyarse en ellas para estructurar una clase es fundamental y bastante útil, permite ir complejizando su conocimiento de manera gradual, evitando la confusión y la apatía hacia los temas explicados.

\section{REFERENCIAS}

Ausubel, D. (1983). Teoría del aprendizaje significativo. Fascículos de CEIF, 1-10.

Beltrán y castro (2016). Ideas previas del concepto reacción química y su relación con conceptos biológicos en estudiantes de grado noveno. Memorias V Congreso Nacional de Investigación en Educación en Ciencias y Tecnología. Neiva, Colombia.

Denzin, Norman K. \& Lincoln, Yvonna S. (1994). Introduction: Entering the field of qualitative research. En Norman K. Denzin \& Yvonna S. Lincoln (Eds.), Handbook of qualitative research (pp.1-17). Thousand Oaks, California: Sage.

González Puentes, F., \& García-Pérez, F. F. (2014). Las concepciones de los alumnos sobre el medio urbano y sus implicaciones didácticas. Un estudio en Bogotá. Didáctica Geográfica, 41-60.

Giordan, A. (2000). La enseñanza de las ciencias. Planteamientos en educación. Santafé de Bogotá, Colombia: Escuela pedagógica experimental.

Luna Pérez, M., \& Solís Ramírez, E. (1997). Las ideas previas del alumnado en Ciencias: una recopilación sobre los núcleos de contenidos del primer ciclo de la ESO. Título 
Bio - grafía. Escritos sobre la Biología y su Enseñanza. ISSN 2027-1034

Edición Extraordinaria. p.p. 1026 - 1033

Memorias del IX Encuentro Nacional de Experiencias en Enseñanza de la Biología y la Educación Ambiental. IV Congreso Nacional de Investigación en Enseñanza de la Biología.

abierto: revista del CEP de Sevilla, (3), 63-74.

Moreira, M. 1993. Aprendizaje significativo: un concepto subyacente. Instituto de Física, UFRGS. Brasil.

Olave, Y \& Martín, G (2015). Análisis de las concepciones sobre el concepto de sistema nervioso en estudiantes de grado noveno (901) del colegio Antonio Nariño I.E.D. Revista Bio-grafias (2017). 11pp

Pozo, J. I. (1996). Las ideas del alumnado sobre la ciencia: de dónde vienen, a dónde van... y mientras tanto qué hacemos con ellas. Alambique: Didáctica de las ciencias experimentales. Alambique. [Versión electrónica]. Revista Alambique 7

Salazar, L (2013). Enseñanza aprendizaje del concepto de sistema nervioso en estudiantes de básica secundaria. Universidad Nacional de Colombia Facultad, de Ciencias Exactas y Naturales Manizales, Colombia. $121 \mathrm{pp}$. 